\title{
A Systematic Review of the Correlation Between Web-based Query and Outbreak of Emerging Infectious Diseases and Meta-analysis of Influenza- like Illnesses
}

\section{Minh-Duc Nguyen-Tran}

Faculty of medicine, University of Medicine and Pharmacy, Ho Chi Minh City, Vietnam

\section{Nu-Vuong Nguyen-Tran}

Faculty of Medicine, Vietnam National University Ho Chi Minh City

Nguyen Nhu Do Phuc

Department of Epidemiology, Institute of Public Health Ho Chi Minh City, Vietnam

Huu Loc Thai Tran

School of medicine, Viet Nam National University, Ho Chi Minh City, Vietnam

\section{Ahmad Helmy Zayan}

Department of Otolaryngology, Menoufia University, Menoufia, Egypt

\section{Ahmed Saber Abdelrahman}

Faculty of Medicine, Misr University for Science and Technology, Giza, Egypt

\section{Thao Thanh Vu}

School of Health and Biomedical Science, RMIT University, Australia

\section{Khuong An Luu Nguyen}

Ho Chi Minh City University of Medicine and Pharmacy Faculty of Medicine

\section{Trang Thi Nguyen}

Quy Nhon University

\section{Y Nhi Le Vo}

Ho Chi Minh City University of Medicine and Pharmacy

Nhat Minh Nguyen Chu

School of Medicine, Vietnam National University, Ho Chi Minh City, Vietnam

Quynh Thuy Huong Tran

School of Medicine, Vietnam National University, Ho Chi Minh City, Vietnam

\section{My Hoan Pham}

Ho Chi Minh City University of Medicine and Pharmacy

\section{Hai Ngoc Vu}

Ho Chi Minh City University of Medicine and Pharmacy

Nguyen Tien Huy ( $\nabla$ tienhuy@nagasaki-u.ac.jp) 


\section{Research article}

Keywords: Infectious diseases, ILI, Influenza-like illnesses, Outbreaks, Systematic review, Web-based queries

Posted Date: August 31st, 2020

DOI: https://doi.org/10.21203/rs.3.rs-66084/v1

License: (c) (1) This work is licensed under a Creative Commons Attribution 4.0 International License. Read Full License 


\section{A systematic review of the correlation between web-based query and outbreak of emerging infectious diseases and meta-analysis of influenza-like illnesses}

Nguyen Tran Minh Duc ${ }^{1,2}$, Nguyen Tran Nu Vuong ${ }^{2,3}$, Do Phuc Nhu Nguyen ${ }^{2,7, \square}$, Tran Thai Huu Loc ${ }^{2,3}$, Ahmad Helmy Zayan ${ }^{2,4}$, Ahmed Saber Abdelrahman ${ }^{2,5}$, Thao Thanh $\mathrm{Vu}^{2,6}$, Luu Nguyen An Khuong ${ }^{1,2}$, Nguyen Thi Trang ${ }^{2,8}$, Vo Le Y Nhi ${ }^{1,2}$, Chu Nguyen Nhat Minh $^{2,3}$, Tran Thuy Huong Quynh ${ }^{2,3}$, Pham Hoan My ${ }^{1,2}$, Vu Ngoc Hai ${ }^{1,2}$, Nguyen Tien Huy $9.10,11^{*}$

\section{$\underline{\text { Affiliations: }}$}

${ }^{1}$ Faculty of Medicine, University of Medicine and Pharmacy, Ho Chi Minh City, Vietnam

${ }^{2}$ Online research Club (http://www.onlineresearchclub.org/).

${ }^{3}$ School of Medicine, Viet Nam National University Ho Chi Minh City, Vietnam

${ }^{4}$ Department of Otolaryngology, Menoufia University, Menoufia, Egypt

${ }^{5}$ Faculty of Medicine, Misr University for Science and Technology, Giza, Egypt.

${ }^{6}$ School of Health and Biomedical Science, RMIT University, Australia.

${ }^{7}$ Department of Epidemiology, Institute of Public Health Ho Chi Minh City, Vietnam

${ }^{8}$ Quy Nhon City Hospital, Binh Dinh Province, Ho Chi Minh City, Viet Nam

${ }^{9}$ Institute of Research and Development, Duy Tan University, Da Nang 550000, Vietnam.

${ }^{11}$ Department of Clinical Product Development, Institute of Tropical Medicine (NEKKEN), Leading Graduate School Program, and Graduate School of Biomedical Sciences, Nagasaki University, 1-124 Sakamoto, Nagasaki 852-8523, Japan 
$\neg$ Authors equally contributed the work.

*Corresponding author: Nguyen Tien Huy, Institute of Research and Development, Duy Tan University, Da Nang 550000, Vietnam (E-Mail: nguyentienhuy4@ duytan.edu.vn).

\section{Emails:}

NTMD: minhduc1298@gmail.com (ORCID: 0000-0002-9333-7539)

NTNV: ntnvuong.y2016@ medvnu.edu.vn (ORCID: 0000-0003-1167-7650)

DPNN: dophucnhunguyen@iph.org.vn (ORCID: 0000-0002-7644-4658)

TTHL: tthloc.stu13@ medvnu.edu.vn (ORCID: 0000-0001-5518-0887)

AHZ: ahmad_zayan@yahoo.com (ORCID: 0000-0003-2581-0459)

ASA: dr.ahmedsaber@icloud.com (ORCID: 0000-0003-2049-6085)

TTV: thaovuthanhthao@gmail.com (ORCID: 0000-0003-0980-0293)

LNAK: louis.lnak1410@gmail.com (ORCID: 0000-0002-8558-5946)

NTT: trangnguyenqn.dr@gmail.com (ORCID: 0000-0003-4676-0905)

VLYN: vly.nhi1995@gmail.com (ORCID: 0000-0002-3738-623X)

CNNM: cnnminh.y2016@ medvnu.edu.vn (ORCID: 0000-0002-3229-636)

TTHQ: tranquynh.su@ gmail.com (ORCID: 0000-0002-6077-4783)

PHM: hoanmy.yds@gmail.com (ORCID: 0000-0003-1686-1465)

VNH: dr.vungochai@gmail.com (ORCID: 0000-0002-3202-8871)

NTH: nguyentienhuy4@ duytan.edu.vn (ORCID: 0000-0002-9543-9440) 


\section{$\underline{\text { Abstract }}$}

Background: Emerging infectious diseases (EIDs) are among the widespread everchanging threats to public health. Web-based queries using information gathered from social media can enhance global syndromic surveillance to trace EIDs activity. This systematic review aimed to investigate the correlation of web-based queries to outbreak of EIDs.

Methods: Nine electronic databases were systematically searched and updated in August 2018 including; PubMed, Virtual Health Library (VHL), WHO Global Health Library (GHL), Scopus, ISI, Google Scholar, POPLINE, and Systems for Information of Grey Literature in Europe (SIGLE), New York Academy of Medicine (NYAM Grey Literature Report). A prior protocol was registered at Prospero (CRD42016038104). In a total five included articles, 47 datasets were included for reviewing. The correlation was assessed through Spearman and Pearson tests using either google trends or number of tweets.

Results: Meta-analysis of influenza-like illness data revealed that correlation was significant $(0.784(0.743-0.820,0.964(0.918-0.985)$ for both Spearman and Pearson tests respectively.

Conclusions: Web-based surveillance systems could serve as a good method in predicting events of EIDs.

\section{$\underline{\text { Keywords }}$}

Infectious diseases; ILI; Influenza-like illnesses; Outbreaks; Systematic review; Web-based queries 


\section{Background:}

Emerging infectious diseases (EIDs) have been escalating in the past 20 years and threatening to over 17 million deaths worldwide in public health every year ${ }^{1}$. During ongoing emerging infectious diseases, prediction using search query data provides an optimal robust and sensitive solution for rapidly detecting the distribution of diseases and other health conditions over time, forecasting disease outbreaks in different geographical areas and controlling an outbreak ${ }^{2}$. This query system proved its power in most recent epidemics, such as influenza epidemics ${ }^{3}$. Traditional surveillance systems reply on both virological and clinical data, then national and regional data is published on a weekly basis, frequently with a 1-2 week reporting lag ${ }^{3}$. In developing countries, surveillance for such detection is costly, and lack the public health framework to determine outbreaks at their earliest stages. Furthermore, the internet has freely available web-based sources of information and subsequently faster detection at low cost. Eighty percent of American internet users, or about 113 million adults, are believed to search online for health information about specific diseases or medical conditions ${ }^{4}$, millions of people worldwide use online to search for health-related information each day, making web-based queries a valuable source of information on recent health trends ${ }^{5}$. This calls into question about the precision of these queries on the detection and estimation of the global EIDs burden. Therefore, this study aims to investigate the correlation of web-based queries to the outbreak of EIDs.

\section{Methods}




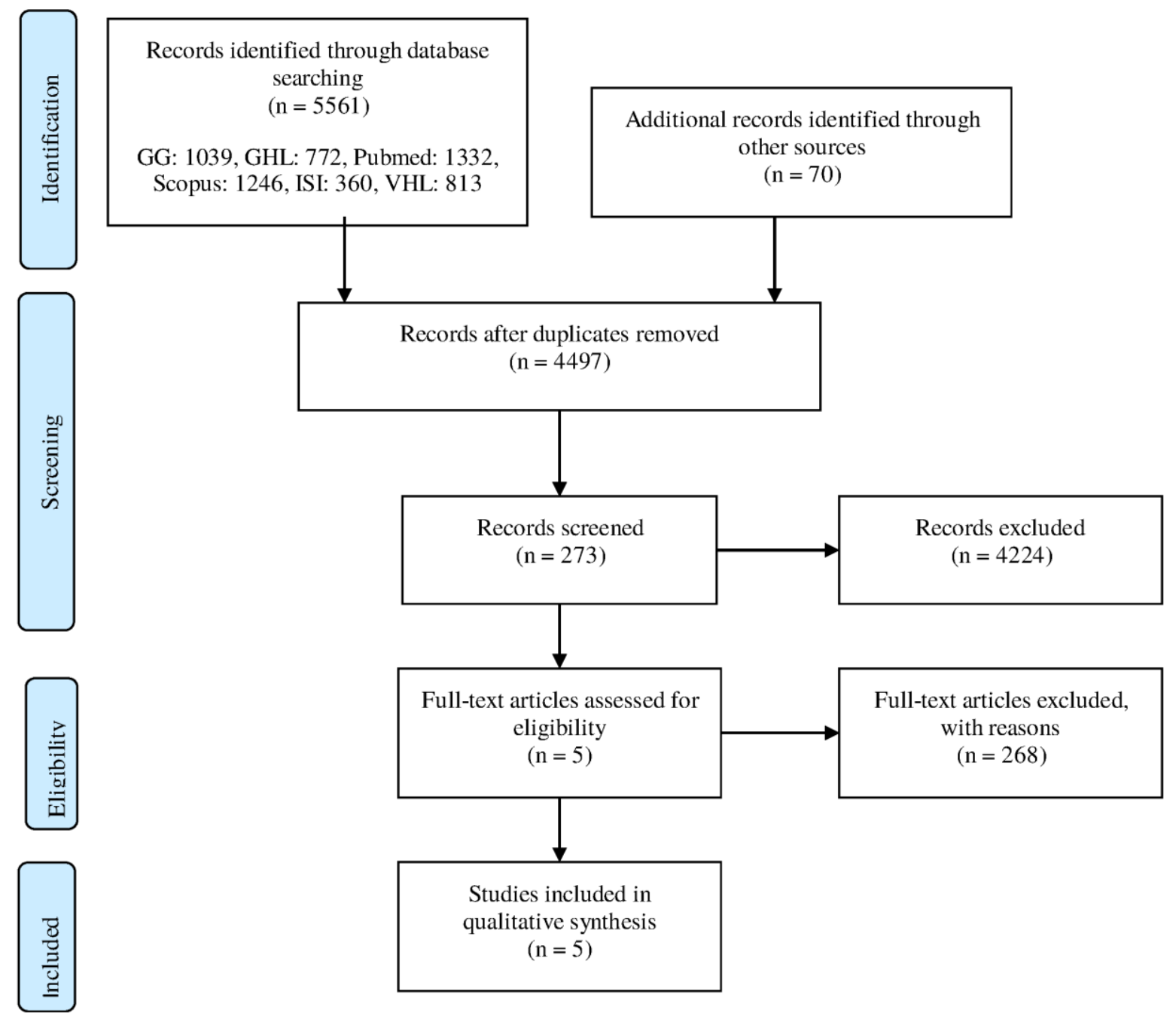

Figure 1. Flow diagram of study design
No. ID

$\begin{array}{llll}\text { Country } & \begin{array}{l}\text { Year of } \\ \text { collecting } \\ \text { data }\end{array} & \begin{array}{l}\text { Web } \\ \text { query }\end{array} & \text { Disease } \\ & & \end{array}$
Sample Correlation
size coefficient

\section{Spearman's correlation}




\begin{tabular}{|c|c|c|c|c|c|c|c|}
\hline \multirow[t]{19}{*}{1} & \multirow{19}{*}{ Milinovich/2014 6} & \multirow[t]{19}{*}{ Australia } & 2009- & \multirow[t]{19}{*}{ Google } & Pneumococcal disease (invasive) & 8096 & .882 \\
\hline & & & 2013 & & Gonococcal infection & 55068 & .847 \\
\hline & & & & & Ross River virus infection & 23000 & .811 \\
\hline & & & & & Varicella zoster (Unspecified) & 37204 & .783 \\
\hline & & & & & Influenza (laboratory confirmed) & 165797 & .775 \\
\hline & & & & & Dengue virus infection & 6392 & .754 \\
\hline & & & & & Chlamydia infection & 362141 & 699 \\
\hline & & & & & Pertussis & 136494 & .814 \\
\hline & & & & & Varicella zoster (Shingles) & 17504 & .783 \\
\hline & & & & & Varicella zoster (Chickenpox) & 8843 & 689 \\
\hline & & & & & Hepatitis C (unspecified) & 48731 & 617 \\
\hline & & & & & Barmah Forest virus infection & 10327 & .683 \\
\hline & & & & & Meningococcal disease (invasive) & 1067 & .586 \\
\hline & & & & & Hepatitis B (unspecified) & 31965 & .542 \\
\hline & & & & & Measles & 635 & .534 \\
\hline & & & & & Murray Valley encephalitis virus & 23 & .544 \\
\hline & & & & & infection & 14297 & .449 \\
\hline & & & & & Cryptosporidiosis & 239 & .436 \\
\hline & & & & & Chikungunya virus infection & & \\
\hline \multirow[t]{28}{*}{2} & \multirow{28}{*}{ Aslam/2014 9} & \multirow[t]{28}{*}{ USA } & 2013- & \multirow[t]{28}{*}{ Twitter } & Sentinel-provided ILI rates in & 3813 & 0.100 \\
\hline & & & 2014 & & Boston & 5116 & 0.640 \\
\hline & & & & & Sentinel-provided ILI rates in & 1497 & 0.600 \\
\hline & & & & & Chicago & 1034 & -0.240 \\
\hline & & & & & Sentinel-provided ILI rates in & 1942 & 0.690 \\
\hline & & & & & Cleveland & 2195 & 0.760 \\
\hline & & & & & Sentinel-provided ILI rates in & 1236 & 0.850 \\
\hline & & & & & Columbus & 1630 & 0.830 \\
\hline & & & & & Sentinel-provided ILI rates in & 12632 & 0.550 \\
\hline & & & & & Denver & 1808 & 0.880 \\
\hline & & & & & Sentinel-provided ILI rates in & 3813 & 0.610 \\
\hline & & & & & Detroit & 5116 & 0.800 \\
\hline & & & & & Sentinel-provided ILI rates in Fort & 1497 & 0.750 \\
\hline & & & & & Worth & 1034 & 0.870 \\
\hline & & & & & Sentinel-provided ILI rates in & 1808 & 0.880 \\
\hline & & & & & Nashville-Davidson & 2941 & 0.820 \\
\hline & & & & & Sentinel-provided ILI rates in New & & \\
\hline & & & & & York & & \\
\hline & & & & & Sentinel-provided ILI rates in San & & \\
\hline & & & & & Diego & & \\
\hline & & & & & Emergency department ILI rates in & & \\
\hline & & & & & Boston & & \\
\hline & & & & & Emergency department ILI rates in & & \\
\hline & & & & & Chicago & & \\
\hline & & & & & Emergency department ILI rates in & & \\
\hline & & & & & Cleveland & & \\
\hline & & & & & Emergency department ILI rates in & & \\
\hline & & & & & Columbus & & \\
\hline
\end{tabular}




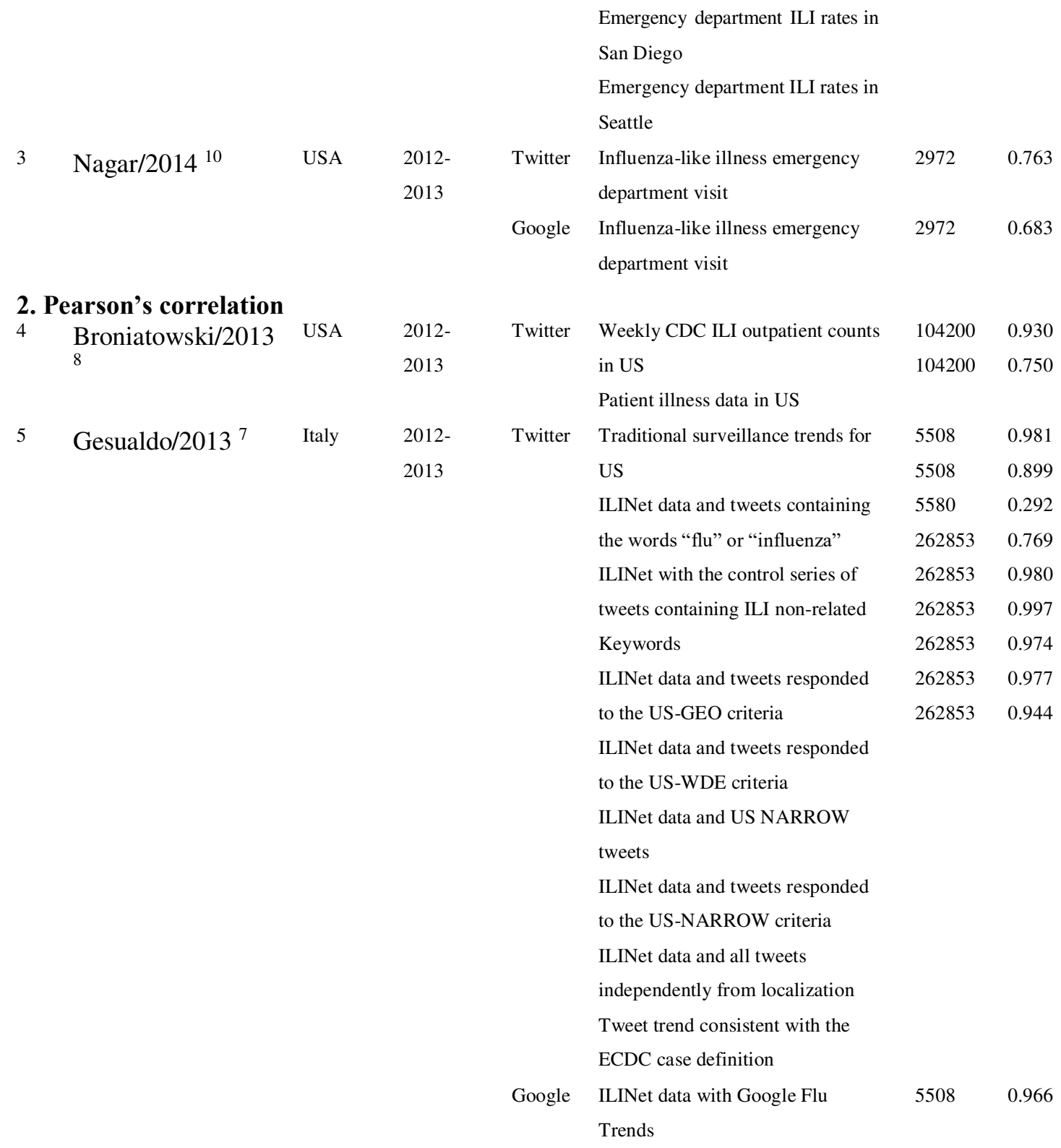

Table 1. Basic characteristics of the included studies.

We included original articles reporting the correlation of the web search query and number or prediction of cases and the correlation coefficient values such as $\mathrm{R}^{2}$, Pearson or Spearman's coefficient without restriction in human (Supplementary Table 1). In February 2016, nine electronic databases were searched carefully to identify relevant articles and an update was conducted in February 2019. We used the search term which is presented in Supplementary Table 2. A manual search - of relevant articles, related searches, and 
citations from the included articles, PubMed, and Google Scholar, respectively- was performed by three independent reviewers. Search results were imported into Endnote X7 (Thompson Reuter, CA, USA) to remove duplicates. Three independent reviewers screened the title and abstract by using the pre-determined eligibility criteria. If the title and abstract did not provide enough information, full-text reading is required. In the case of disagreement, the consensus was reached by discussion among reviewers or guidance from the supervisors. The standardized template was developed through a pilot extraction with the two most relevant references. Extracted data included basic and special information which are presented in (Table 1). For the meta-analysis, we used the correlation between Google Trends or number of tweets and the best fit model of representative influenza-like illness data (emergency department visits, influenza illness surveillance program data (ILINet), Centers for Disease Control and Prevention counts (CDC), and laboratoryconfirmed cases). The summary estimates with $95 \%$ Confidence Interval (CI) of each correlation coefficient would be calculated using the R statistical software 3.5 Heterogeneity would be assessed through the Chi-squared-based Q test or the I2 method. Fixed-effects model would be applied if no evident significant heterogeneity $(\mathrm{P}<0.05$., I2 $<50 \%)$. Otherwise, the random-effects model was used ${ }^{6}$. All P values were two-sided and were considered statistically significantly less than 0.05 .

\section{$\underline{\text { Results }}$}

Among the 5674 records were found, distributed among the nine databases and after removing the duplicated articles, 4478 remained for analysis of the titles and abstracts. During the phase of screening the titles and abstract, four thousand and five studies were 
excluded, and thus 273 studies were eligible for analysis of the complete text. After excluding 268 articles, five articles were eligible for review (Figure 1 and Table 1).

The included articles were published between 2013-2014, the most included infectious diseases were influenza and influenza-like illnesses. Of the five included studies, three were conducted in the United States, one in Italy and one in Australia. The study period varied with a duration ranging from 5 months to 9 years. Google trends were investigated in two articles (Millinovich et. $\mathrm{al}^{6}$, Gesualdo et. $\mathrm{al}^{7}$ ) and Twitter data were investigated in the rest of articles (Broniatowski et.al ${ }^{8}$, Aslam et $\mathrm{al}^{9}$, Nagar et $\mathrm{al}^{10}$ )

Spearman correlation was investigated in three articles (Millinovich et. $\mathrm{al}^{6}$, Aslam et $\mathrm{al}^{9}$, Nagar et $\mathrm{al}^{10}$ ) and ranged between -0.24 to 0.88 . Millinovich et $\mathrm{al}^{6}$, investigated correlation google trends and 17 different infectious diseases using time series analysis on monthly notifications. They also concluded the potential applicability of web-based queries for vector-borne disease. In the second article (Aslam et al ${ }^{9}$ ), the weekly number of tweets of influenza/influenza-like illness without URL demonstrated higher accuracy values in predicting laboratory-confirmed cases for most categories compared to URL based ones $(\mathrm{r}=0.93)$. A spatiotemporal time analysis was used in Nagar et $\mathrm{a}^{10}$ and revealed better correlation coefficients for daily flu tweets than daily google search volume (r=0.763 vs $0.683)$ 


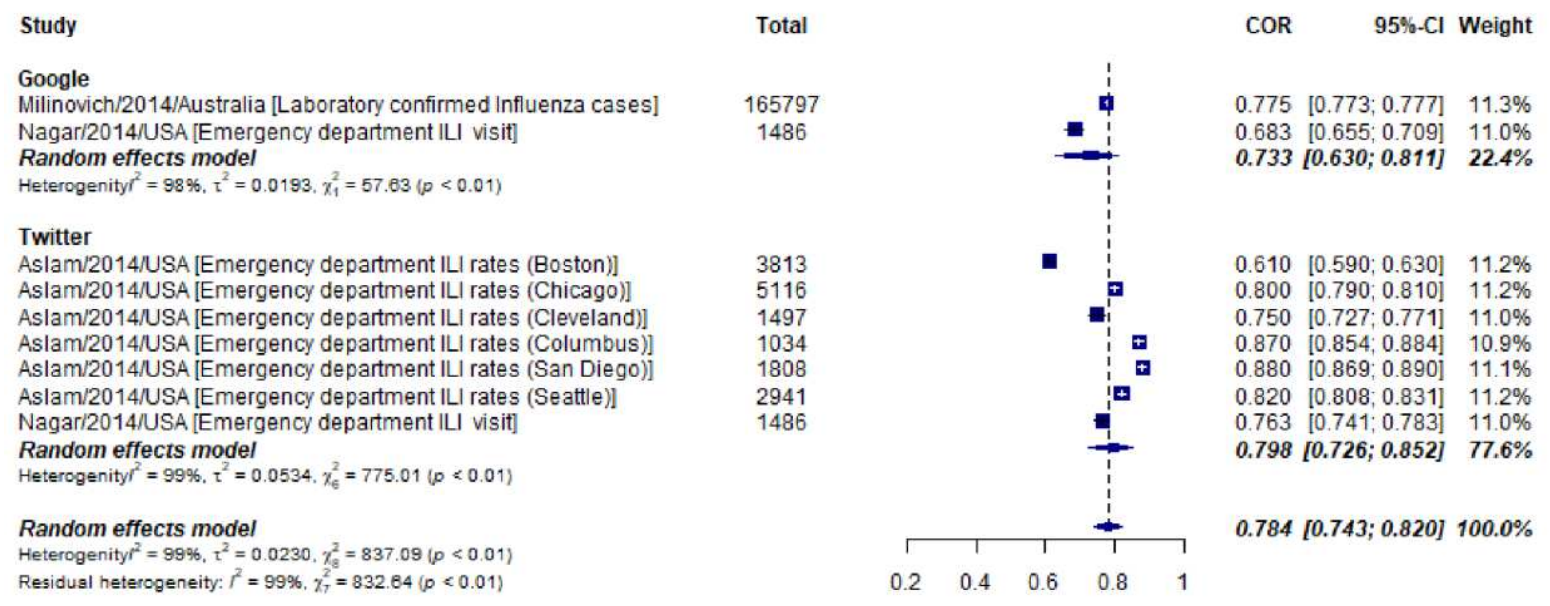

Figure 2. Meta-analysis of spearman correlation of influenza like illness across web-

\section{based queries, ILI=influenza like illness}

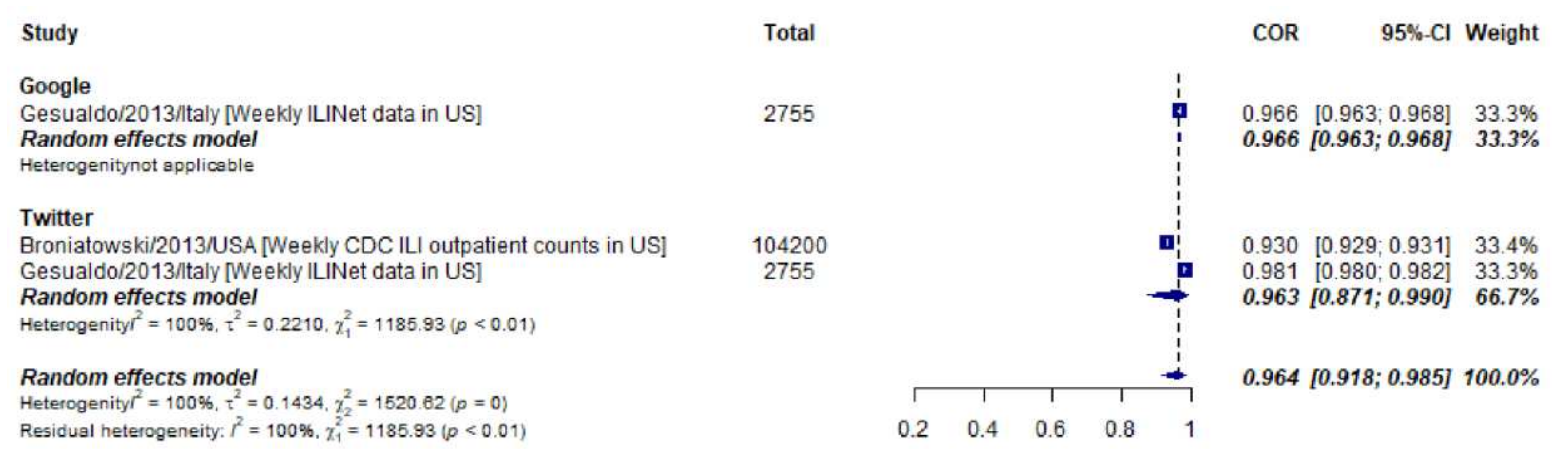

Figure 3. Meta-analysis of Pearson correlation of influenza like illness across web-

\section{based queries}

Meanwhile, Pearson's correlation was studied in the other two articles (Broniatowski et al ${ }^{8}$, Gesualdo et $\mathrm{al}^{7}$ ) and ranged between 0.29 to 0.98 . Broniatowski et al ${ }^{8}$ demonstrated the same significant efficacy of twitter data compared to CDC surveillance data for influenzalike illness patients for a lag of one week(r=0.93). In the same context, Gesualdo et $\mathrm{al}^{7}$, demonstrated a significant correlation between influenza-positive tweets or google trends with ILINet data $(\mathrm{r}=0.98,0.96$ respectively)

Using the random-effects model, a meta-analysis of influenza-like illness data showed that correlation was significant $(0.784(0.743-0.820), 0.964(0.918-0.985)$ for both Spearman and 
Pearson tests respectively (Figure 2, 3). Subgroup analysis for results of Spearman correlation demonstrated better efficacy for twitter data ( 7 datasets, three articles) $(0.798$ (0.726-0.818) vs google trends (two datasets, two articles) $(0.733$ (0.630-0.811). Further subgroup analysis based on a period of data collection revealed that weekly data outperforms both daily and monthly data (0.803 (0.773-0.863), $0.725(0.637-0.795), 0.775$ (0.773-0.777), respectively).

\section{$\underline{\text { Discussion }}$}

We investigated how well web queries submitted to the social media mimic the results from other systems for emerging infectious diseases (EIDs) surveillance. For the most common advantages, the web-based query could help track and predict ongoing pandemics for the most popular infectious diseases worldwide, and therefore planning for better prophylaxis and prevention ${ }^{11}$. As well, when these data were combined with other applications such as air traffic data, the query could enhance tremendously to the prediction of the spread of certain infectious diseases ${ }^{12}$.

Through a meta-analysis of influenza/influenza-like illness data, we found a significant correlation at both Spearman and Pearson tests. Moreover, our results demonstrated better prediction values of twitter data versus google search, these were supported by results of Aslam et al. ${ }^{9}$, Broniatowski et al. ${ }^{8}$, and Nagar et al ${ }^{7}$. Regarding a period of data collection for trend analysis, we also demonstrated the better performance of weekly based model data. This can be explained by the temporal resolution of data if it was used on a monthly basis. ${ }^{6}$ From a different perspective, other internet-based surveillance, as in the study of Milinovich et al., was not only used in tracking and predicting influenza prevalence but also in the 
management of other infectious diseases such as dengue fever. Through using a wide range of specific search terms, 17 infectious diseases (26.6\%) were found to be significantly correlated $^{6}$. They also recommended that search terms that present highly significant correlation should be kept for re-using as they can help in providing a quicker response on future emerging disease management

However, social media such as Twitter or Google can have a few limitations. A significant one is that we could not collect demographic data like age, sex and racial characteristics of patients via tweets, which could cause difficulty for the public health sector to make a response $^{9}$. Another concern is that Twitter is used mainly in one group of the population, for example, people living in metropolitan areas, which may cause unavoidable bias in data retrieving as the data cannot represent the characteristic of the whole population ${ }^{7}$. Twitter also required a large human resource to classify the tweets, and therefore implied the potential of human error ${ }^{10}$. In addition, the search engine does not show the IP-address which could show us the specific location of the users; therefore, it is only possible to track the epidemic on the national scale ${ }^{13}$. But using the data with precise information connected to individuals could violate their privacy. Fifthly, because of the huge synonyms of query terms, the web-based tool may underestimate the real disease activity since a lot of terms may not be gathered ${ }^{14}$. Sixthly, forecasting health and disease-related phenomena have a very high chance of false-positive because people perceive their health status is very subjective. Thus, data sources must be carefully collected before all analyses ${ }^{15}$. Finally, due to the limited number of included articles, our study could hardly reflect accurately the clear correlation between web-based query prediction results and government announcement. Hence, we are looking forward to more research on how certain factors could alter 
predictive results and in this way, developing tools to filter those factors in the attempt to complete the capacity of prediction thanks to web-based queries.

\section{Conclusions}

In conclusion, web-based surveillance systems could serve as a good method in predicting events of emerging infectious diseases.

\section{List of abbreviations}

EIDs: Emerging infectious diseases

ILI: Influenza-like illnesses

\section{Declarations}

Ethics approval and consent to participate:

Not applicable

\section{Consent for publication:}

Not applicable

\section{Availability of data and material:}

All data generated or analysed during this study are included in this published article.

\section{Competing interests:}

The authors declare that they have no competing interests. 


\section{Funding:}

This work was supported in part by a "Grant-in-Aid for Scientific Research (B)" (16H05844, 2016-2019 for Nguyen Tien Huy) from Ministry of Education, Culture, Sports, Science and Technology (MEXT) of Japan. The funders had no role in the study design, data collection and analysis, decision to publish or preparation of the manuscript.

\section{Authors' contributions:}

NTMD, VNH, NTH: conceptualization, supervision; NTMD, NTNV, DPNN, TTV, LNAK, NTT, VLYN, CNNM, TTHQ, PHM: screening and data collecting, formal analysis and writing-original draft preparation; TTHL, AHZ, ASA: methodology, formal analysis, writing-reviewing, editing; and software. The authors read and approved the final manuscript.

\section{Acknowledgements:}

Not applicable.

\section{Supplementary materials}

\section{Supplementary Table 1. PRISMA 2009 Checklist.}

$\begin{array}{llll}\text { Section/topic } & \# & \text { Checklist item } & \begin{array}{l}\text { Reported } \\ \text { on page \# }\end{array} \\ \text { Title } & 1 & \text { Identify the report as a systematic review, meta-analysis, or both. } & \text { Page 1 } \\ \text { Structured } & 2 & \begin{array}{l}\text { Provide a structured summary including, as applicable: } \\ \text { summary }\end{array} & \text { background; objectives; data sources; study eligibility criteria, } \\ & & \text { participants, and interventions; study appraisal and synthesis } \\ & & \\ & & \text { methods; results; limitations; conclusions and implications of key } \\ & \text { findings; systematic review registration number. }\end{array}$



already known.

Objectives 4 Provide an explicit statement of questions being addressed with reference to participants, interventions, comparisons, outcomes, and study design (PICOS).

Protocol and

5 Indicate if a review protocol exists, if and where it can be registration accessed (e.g., Web address), and, if available, provide registration information including registration number.
Eligibility 6 Specify study characteristics (e.g., PICOS, length of follow-up) Page 6 criteria and report characteristics (e.g., years considered, language, publication status) used as criteria for eligibility, giving rationale.

Information

7 Describe all information sources (e.g., databases with dates of

sources coverage, contact with study authors to identify additional studies) in the search and date last searched.

Search

8 Present full electronic search strategy for at least one database, including any limits used, such that it could be repeated.

Study

9 State the process for selecting studies (i.e., screening, eligibility,

selection included in systematic review, and, if applicable, included in the meta-analysis).

Data

10 Describe method of data extraction from reports (e.g., piloted

collection

process forms, independently, in duplicate) and any processes for obtaining and confirming data from investigators.

Data items

11 List and define all variables for which data were sought (e.g., PICOS, funding sources) and any assumptions and simplifications made.

Risk of bias

12 Describe methods used for assessing risk of bias of individual in individual studies (including specification of whether this was done at the studies study or outcome level), and how this information is to be used in any data synthesis.

Summary

13 State the principal summary measures (e.g., risk ratio, difference measures Synthesis of in means).

results

14 Describe the methods of handling data and combining results of studies, if done, including measures of consistency (e.g., I2) for each meta-analysis. 
Risk of bias

across studies

Additional

analyses

Study

selection

Study

characteristics

Risk of bias

within studies

Results of

individual

studies

Synthesis of

results

Risk of bias

across studies

Additional

analysis

Summary of

evidence

Limitations

Conclusions

26 Provide a general interpretation of the results in the context of other evidence, and implications for future research.

Funding

15 Specify any assessment of risk of bias that may affect the

Page 7 cumulative evidence (e.g., publication bias, selective reporting within studies).

16 Describe methods of additional analyses (e.g., sensitivity or subgroup analyses, meta-regression), if done, indicating which were pre-specified.

17 Give numbers of studies screened, assessed for eligibility, and Page 7 included in the review, with reasons for exclusions at each stage, ideally with a flow diagram.

18 For each study, present characteristics for which data were extracted (e.g., study size, PICOS, follow-up period) and provide the citations.

19 Present data on risk of bias of each study and, if available, any outcome level assessment (see item 12).

20 For all outcomes considered (benefits or harms), present, for each study: (a) simple summary data for each intervention group (b) effect estimates and confidence intervals, ideally with a forest plot.

21 Present results of each meta-analysis done, including confidence intervals and measures of consistency.

22 Present results of any assessment of risk of bias across studies (see Item 15).

23 Give results of additional analyses, if done (e.g., sensitivity or subgroup analyses, meta-regression [see Item 16]).

24 Summarize the main findings including the strength of evidence for each main outcome; consider their relevance to key groups (e.g., healthcare providers, users, and policy makers).

25 Discuss limitations at study and outcome level (e.g., risk of bias), and at review-level (e.g., incomplete retrieval of identified research, reporting bias).

27 Describe sources of funding for the systematic review and other support (e.g., supply of data); role of funders for the systematic review.
Page 12

Page 5

Page 16

Page 17

Page 7

Page 17

Page 12

Page 12

Page 13 


\title{
Supplementary Table 2. Search terms of each included database.
}

\author{
Database Result Search term
}

PubMed 1332 (outbreaks or outbreak or epidemic or pandemic or dengue or infectious or influenza or flu or zika or foodborne or "food-borne" or waterborne or "waterborne" or "mosquito-borne" or vectorborne or "vector-borne" ) and (forecasting or forecast or prediction or predictions or predict or predictive or predicted or "early warning" or monitor or monitoring) and (online or internet or web or google or googling or baidu or query or queries or infodemiology or "digital disease detection" or infoveillance or "real-time disease surveillance" or "syndromic surveillance" or "social media" or "social network" or Twitter or Facebook or Instagram) and (relationship or relation or correlation or correlations or correlated or correlate or Pearson or Spearman or (sensitivity and specificity))

ISI:

360 (outbreaks or outbreak or epidemic or pandemic or dengue or infectious or influenza or flu or zika or foodborne or "food-borne" or waterborne or "waterborne" or "mosquito-borne" or vectorborne or "vector-borne" ) and (forecasting or forecast or prediction or predictions or predict or predictive or predicted or "early warning" or monitor or monitoring) and (online or internet or web or google or googling or baidu or query or queries or infodemiology or "digital disease detection" or infoveillance or "real-time disease surveillance" or "syndromic surveillance" or "social media" or "social network" or Twitter or Facebook or Instagram) and (relationship or relation or correlation or correlations or correlated or correlate or Pearson or Spearman or (sensitivity and specificity))

Scopus $\quad 1246$ (outbreaks or outbreak or epidemic or pandemic or dengue or infectious or influenza or flu or zika or foodborne or "food-borne" or waterborne or "waterborne" or "mosquito-borne" or vectorborne or "vector-borne" ) and (forecasting or forecast or prediction or predictions or predict or predictive or predicted or "early warning" or monitor or monitoring) and (online or internet or web or google or googling or baidu or query or queries or infodemiology or "digital disease detection" or infoveillance or "real-time disease surveillance" or 
"syndromic surveillance" or "social media" or "social network" or Twitter or Facebook or Instagram) and (relationship or relation or correlation or correlations or correlated or correlate or Pearson or Spearman or (sensitivity and specificity))

SIGLE $\quad 0$ (outbreaks or outbreak or epidemic or pandemic or dengue or infectious or influenza or flu or zika or foodborne or "food-borne" or waterborne or "waterborne" or "mosquito-borne" or vectorborne or "vector-borne" ) and (forecasting or forecast or prediction or predictions or predict or predictive or predicted or "early warning" or monitor or monitoring) and (online or internet or web or google or googling or baidu or query or queries or infodemiology or "digital disease detection" or infoveillance or "real-time disease surveillance" or "syndromic surveillance" or "social media" or "social network" or Twitter or Facebook or Instagram) and (relationship or relation or correlation or correlations or correlated or correlate or Pearson or Spearman or (sensitivity and specificity))

NYAM $\quad 0$ (outbreaks or outbreak or epidemic or pandemic or dengue or infectious or influenza or flu or zika or foodborne or "food-borne" or waterborne or "waterborne" or "mosquito-borne" or vectorborne or "vector-borne") and (forecasting or forecast or prediction or predictions or predict or predictive or predicted or "early warning" or monitor or monitoring) and (online or internet or web or google or googling or baidu or query or queries or infodemiology or "digital disease detection" or infoveillance or "real-time disease surveillance" or "syndromic surveillance" or "social media" or "social network" or Twitter or Facebook or Instagram) and (relationship or relation or correlation or correlations or correlated or correlate or Pearson or Spearman or (sensitivity and specificity))

POPLINE $\quad 0 \quad$ (outbreaks or outbreak or epidemic or pandemic or dengue or infectious or influenza or flu or zika or foodborne or "food-borne" or waterborne or "waterborne" or "mosquito-borne" or vectorborne or "vector-borne" ) and (forecasting or forecast or prediction or predictions or predict or predictive or predicted or "early warning" or monitor or monitoring) and (online or internet or web or google or googling or baidu or query or queries or infodemiology or "digital disease detection" or infoveillance or "real-time disease surveillance" or "syndromic surveillance" or "social media" or "social network" or Twitter or Facebook or Instagram) and (relationship or relation or correlation or 
correlations or correlated or correlate or Pearson or Spearman or (sensitivity and specificity))

VHL 813 (outbreaks or outbreak or epidemic or pandemic or dengue or infectious or influenza or flu or zika or foodborne or "food-borne" or waterborne or "waterborne" or "mosquito-borne" or vectorborne or "vector-borne" ) and (forecasting or forecast or prediction or predictions or predict or predictive or predicted or "early warning" or monitor or monitoring) and (online or internet or web or google or googling or baidu or query or queries or infodemiology or "digital disease detection" or infoveillance or "real-time disease surveillance" or "syndromic surveillance" or "social media" or "social network" or Twitter or Facebook or Instagram) and (relationship or relation or correlation or correlations or correlated or correlate or Pearson or Spearman or (sensitivity and specificity))

GHL 772 (outbreaks or outbreak or epidemic or pandemic or dengue or infectious or influenza or flu or zika or foodborne or "food-borne" or waterborne or "waterborne" or "mosquito-borne" or vectorborne or "vector-borne" ) and (forecasting or forecast or prediction or predictions or predict or predictive or predicted or "early warning" or monitor or monitoring) and (online or internet or web or google or googling or baidu or query or queries or infodemiology or "digital disease detection" or infoveillance or "real-time disease surveillance" or "syndromic surveillance" or "social media" or "social network" or Twitter or Facebook or Instagram) and (relationship or relation or correlation or correlations or correlated or correlate or Pearson or Spearman or (sensitivity and specificity))

GOOGLE 1039 (outbreaks or outbreak or epidemic or pandemic or dengue or infectious or SCHOLAR influenza or flu or zika or foodborne or "food-borne" or waterborne or "waterborne" or "mosquito-borne" or vectorborne or "vector-borne" ) and (forecasting or forecast or prediction or predictions or predict or predictive or predicted or "early warning" or monitor or monitoring) and (online or internet or web or google or googling or baidu or query or queries or infodemiology or "digital disease detection" or infoveillance or "real-time disease surveillance" or "syndromic surveillance" or "social media" or "social network" or Twitter or Facebook or Instagram) and (relationship or relation or correlation or correlations or correlated or correlate or Pearson or Spearman or (sensitivity and specificity)) 


\section{References:}

1. Key KK, DeNoon DJ. Infectious diseases kill over 17 million people a year. AIDS Wkly 1995. 1996;22-27.

2. Eysenbach G. Infodemiology and infoveillance: framework for an emerging set of public health informatics methods to analyze search, communication and publication behavior on the Internet. J Med Internet Res. 2009;11(1):e11.

3. Ginsberg J, Mohebbi MH, Patel RS, Brammer L, Smolinski MS, Brilliant L. Detecting influenza epidemics using search engine query data. Nature. 2009;457(7232):1012.

4. Fox S. Online health research is widespread, but few check the source and date. Medscape Gen Med. 2007;9(1):30.

5. Johnson HA, Wagner MM, Hogan WR, Chapman WW, Olszewski RT, Dowling JN, et al. Analysis of Web access logs for surveillance of influenza. In: Medinfo. 2004. p. $1202-1206$.

6. Milinovich GJ, Avril SM, Clements AC, Brownstein JS, Tong S, Hu W. Using internet search queries for infectious disease surveillance: screening diseases for suitability. BMC Infect Dis. 2014;14(1):690.

7. Gesualdo F, Stilo G, Agricola E, Gonfiantini MV, Pandolfi E, Velardi P, et al. Influenza-Like Illness Surveillance on Twitter through Automated Learning of Naïve Language. PLOS ONE. 2013 Dec 4;8(12):e82489. 
8. Broniatowski DA, Paul MJ, Dredze M. National and Local Influenza Surveillance through Twitter: An Analysis of the 2012-2013 Influenza Epidemic. PLOS ONE. 2013 Dec 9;8(12):e83672.

9. JMIR - The Reliability of Tweets as a Supplementary Method of Seasonal Influenza Surveillance | Aslam | Journal of Medical Internet Research [Internet]. [cited 2019 Apr 10]. Available from: https://www.jmir.org/2014/11/e250

10. Nagar R, Yuan Q, Freifeld CC, Santillana M, Nojima A, Chunara R, et al. A Case Study of the New York City 2012-2013 Influenza Season With Daily Geocoded Twitter Data From Temporal and Spatiotemporal Perspectives. J Med Internet Res. 2014;16(10):e236.

11. Wu S, Weng Y, Ye W, Wang L, Yan Y, Hong R, et al. [Role of syndromic surveillance program in the practice of early detection on disease outbreak]. Zhonghua Liu Xing Bing Xue Za Zhi Zhonghua Liuxingbingxue Zazhi. 2016 Apr;37(4):531-4.

12. Huang Z, Das A, Qiu Y, Tatem AJ. Web-based GIS: the vector-borne disease airline importation risk (VBD-AIR) tool. Int J Health Geogr. 2012 Aug 14;11(1):33.

13. Domnich A, Panatto D, Signori A, Lai PL, Gasparini R, Amicizia D. Age-related differences in the accuracy of web query-based predictions of influenza-like illness. PLoS One. 2015;10(5):e0127754.

14. Hulth A, Rydevik G, Linde A. Web queries as a source for syndromic surveillance. PloS One. 2009;4(2):e4378. 
15. Cook S, Conrad C, Fowlkes AL, Mohebbi MH. Assessing Google Flu Trends Performance in the United States during the 2009 Influenza Virus A (H1N1) Pandemic. PLOS ONE. 2011 Aug 19;6(8):e23610. 
Figures

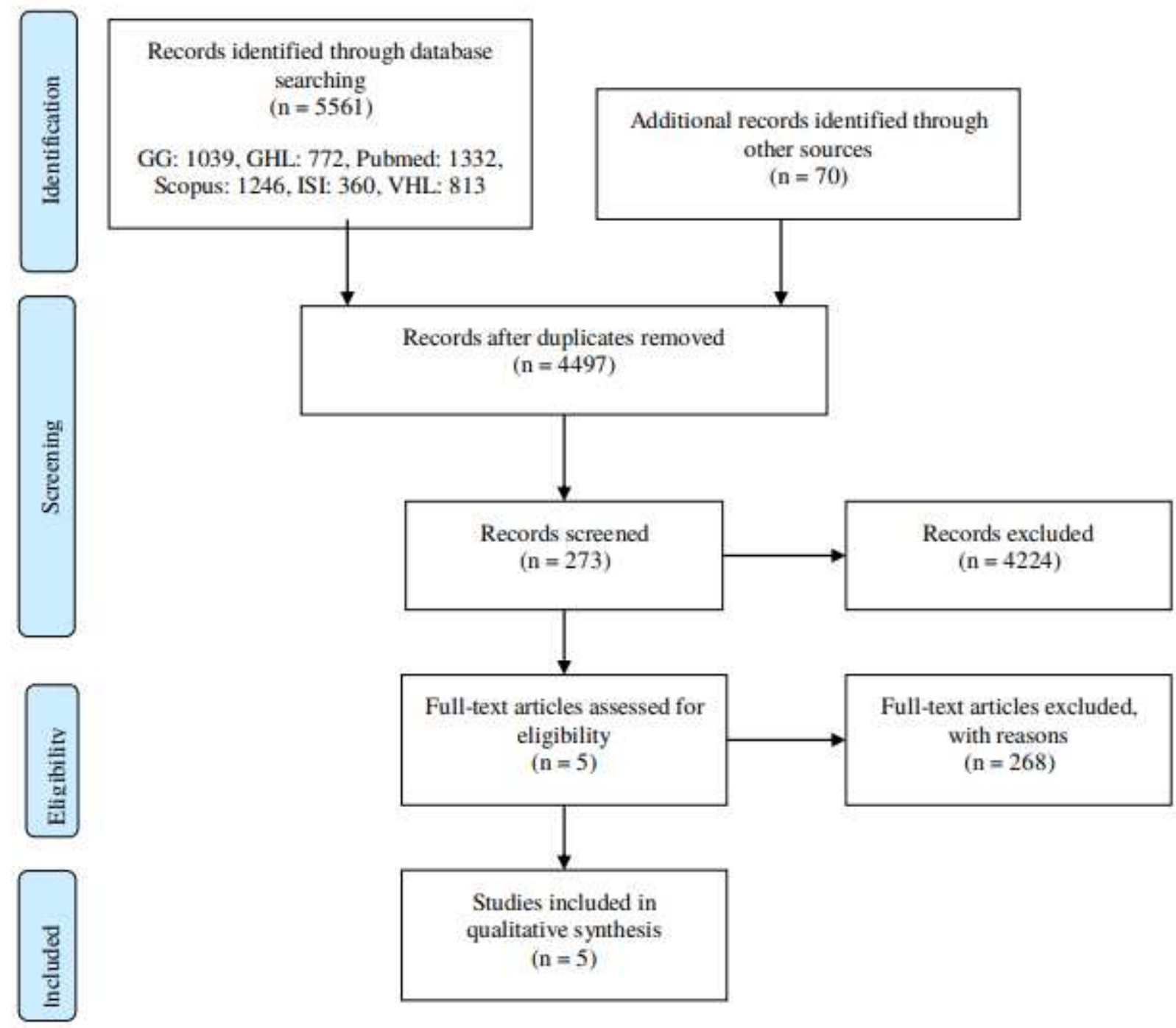

\section{Figure 1}

Flow diagram of study design.

Study

Google

Manowichi2014:Australia Raboratony confirmed infuenza cas es!

Nagsi/2014USA [Emergency department UU visif]

Random effects modol

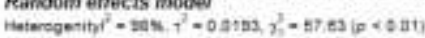

Twitter

Aslam2014USA [Emergency department IL rates (foston]]

Aslam2014USA IEmergenç depantrnentiL fales (Chicago,

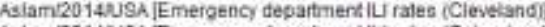

Aslam2014USA [Emergency department IL rates (Columbus)

Astamiz20 14USA |Emergencr departmert IL rates (San Diego)

Aslam20 14USA [Emergenc department IU rates CSeattie?

Nagavi2014USA [Emergency department Li visit!

Random effects modol

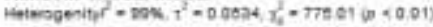

Random effects model

Haturagentipe $=994 . y^{2}=0.0230, y_{2}^{2}=837.09 w<0.01$

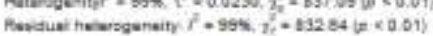

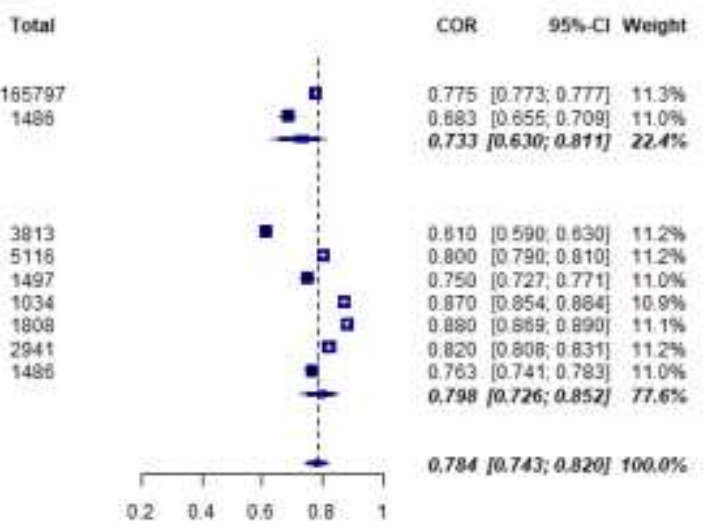


Meta-analysis of spearman correlation of influenza like illness across webbased queries, ILI=influenza like illness


\section{Figure 3}

Meta-analysis of Pearson correlation of influenza like illness across webbased queries, ILINet= influenza illness surveillance program

\section{Supplementary Files}

This is a list of supplementary files associated with this preprint. Click to download.

- Table.pdf 